\section{Hipotiveoidismo Primário Simulando Volumoso Macroadenoma Hipofisário}

\section{RESUMO}

Mulher de 21 anos apresenta história de 2 anos de irregularidade menstrual, com períodos de amenorréia de até 8 meses e ganho ponderal e há 1 ano galactorréia e cefaléia holocraniana. Exames da ocasião: TSH: $1192 \mathrm{mUl} / \mathrm{ml}(0,27-4,2)$; T4T:1,0 $\mu \mathrm{g} / \mathrm{dl}(4,4-11,4)$; T3T: 0,41 ng/ml (0,7$2,1)$; prolactina: $69,2 \mathrm{ng} / \mathrm{ml}(3-20)$. Em serviço de endocrinologia foi confirmado quadro de mixedema acompanhado de galactorréia. Ressonância magnética (RM) de hipotálamo-hipófise mostrou lesão expansiva intra e supra selar com 1,9 x 1,4 x 1,9 cm nos seus maiores diâmetros, determinando compressão e desvio do quiasma óptico. Diante da possibilidade de hiperplasia das células produtoras de TSH, optamos por iniciar o tratamento do hipotireoidismo com levotiroxina. Após 2 meses de tratamento e normalização dos níveis séricos dos hormônios tireoidianos e do TSH, nova RM mostrou hipófise de tamanho normal. A regressão do volume hipofisário após terapia com levotiroxina confirmou a hipótese diagnóstica de hiperplasia hipofisária decorrente do hipotireoidismo primário. Nossos achados reforçam a importância da avaliação dos hormônios tireoideanos e TSH na investigação de aumento de volume hipofisário prevenindo uma cirurgia desnecessária. (Arq Bras Endocrinol Metab 2004;48/3:423-426)

Descritores: Hipotireoisdimo primário; Mixedema; Galactorréia Hiperplasia hipofisária; Macroadenoma hipofisário

\begin{abstract}
Primary Hypothyroidism Mimicking Pituitary Macroadenoma.

A 21 -year-old woman complaining of 8-month amenorrhea associated to weight gain, galactorrhea and frequent headaches, presented for clinical evaluation; her laboratory tests were: TSH: $1192 \mathrm{mUl} / \mathrm{ml}$ (0.27-4.2); TT4: $1.0 \mu \mathrm{g} / \mathrm{dl}$ (4.4-1 1.4I); TT3: $0.41 \mathrm{ng} / \mathrm{ml}$ (0.7-2.1); prolactin: $69.2 \mathrm{ng} / \mathrm{ml}$ (320) and a diagnosis of myxedema associated to galactorrhea was made. A hypothalamic-pituitary magnetic resonance imaging (MRI) showed a suprasellar and intrasellar mass lesion of $1.9 \times 1.4 \times 1.9 \mathrm{~cm}$, determining compression and deviation of the optic chiasm. Due to the possibility of hyperplasia of the TSH-producing cells, treatment of hypothyroidism was initiated with levothyroxine. Two months later, upon normalization of thyroid hormones and TSH levels, a second MRI showed an anatomically normal pituitary gland. Regression of the pituitary mass after treatment with levothyroxine confirmed the hypothesis of pituitary hyperplasia secondary to primary hypothyroidism. Our findings support the importance of determining thyroid function tests during the investigation of pituitary masses and thus avoiding an unnecessary surgery. (Arq Bras Endocrinol Metab 2004;48/3:423-426)
\end{abstract}

Keywords: Primary hypothyroisdim; Myxedema; Galactorrhea; Pituitary

hyperplasia; Pituitary macroadenoma apresentação de

caso

\author{
Carolina C. R. Betônico \\ Ricardo Rodrigues \\ Suzan C. L. Mendonça \\ Paulo Tannus Jorge
}

Departamento de Endocrinologia
da Universidade Federal de
Uberlândia, $M G$
Recebido em 15/12/03

Revisado em 04/05/04

Aceito em 09/05/04 
$\mathbf{O}$ HIPOTIREOIDISMO PRIMÁRIO PODE freqüentemente estar associado com níveis elevados de prolactina, e menos comumente, com amenorréia e galactorréia $(1,2)$. O alargamento da sela túrcica pode ocorrer no hipotireoidismo primário, devido a diminuição crônica dos níveis circulantes de T3 e T4 com hipersecreção do TSH, e subsequente hiperplasia pituitária $(2,3)$. Este aumento do volume hipofisário devido à hiperplasia dos tireotrofos pode ser de difícil diagnóstico, por acompanhar-se de hiperprolactinemia e galactorréia, simulando um adenoma hipofisário (4).

\section{Relato de Caso}

Trata-se de uma paciente de 21 anos, com história de 2 anos de quadro de irregularidade menstrual, com períodos de amenorréia de até 8 meses, acompanhada de ganho ponderal e há $\mathrm{l}$ ano galactorréia e cefaléia holocraniana freqüente. Procurou atendimento médico onde foram solicitados os seguintes exames: TSH: $1192 \mathrm{mUI} / \mathrm{ml}(0,27$ a 4,2$)$; T4T:1,0 $\mu \mathrm{g} / \mathrm{dl}(4,4$ a 11,4); T3T: $0,4 \mathrm{l} \mathrm{ng} / \mathrm{ml}(0,7$ a 2,1$)$; prolactina: 69,2 $\mathrm{ng} / \mathrm{ml}$ (3 a 20). Tomografia computadorizada de hipotálamo-hipófise revelou lesão expansiva intra e supra selar, determinando compressão e desvio do quiasma óptico. Como não apresentava queixas visuais, a campimetria não foi realizada. A paciente foi então encaminhada para o serviço de endocrinologia e confirmado um quadro de mixedema acompanhado de galactorréia. Uma ressonância magnética $(\mathrm{RM})$ de hipotálamo-hipófise confirmou importante aumento de volume hipofisário medindo $1,9 \times 1,4 \times 1,9 \mathrm{~cm}$ nos seus maiores diâmetros (figura 1 ). Diante da possibili-

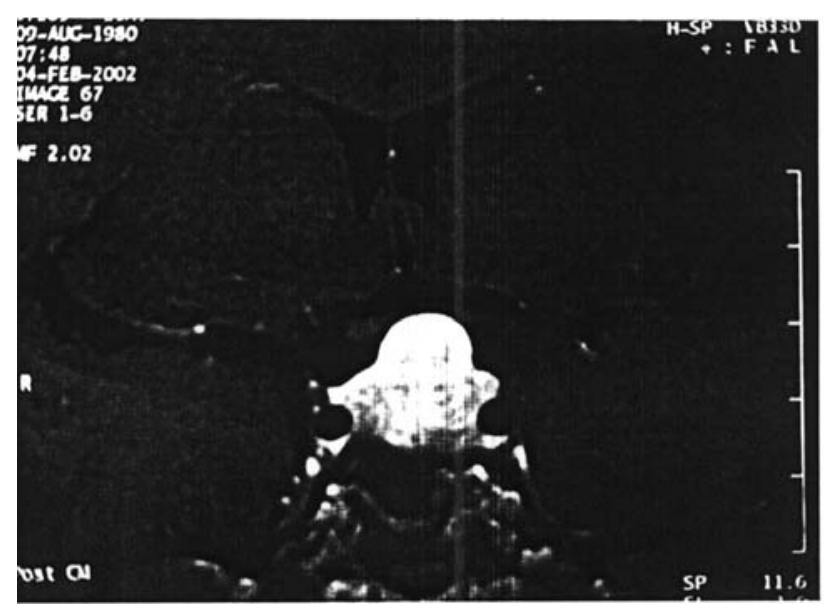

Figura 1. Ressonância magnética (RM) de hipotálamohipófise mostrando importante aumento de volume hipofisário medindo 1,9 × 1,4 × 1,9 cm nos seus maiores diâmetros. dade de tratar-se de uma hiperplasia das células produtoras de TSH, optamos por iniciar o tratamento do hipotireoidismo com levotiroxina sódica. Após 2 meses do início do tratamento e normalização dos níveis séricos dos hormônios tiroidianos e TSH, nova RM mostrou hipófise de tamanho normal (figura 2).

\section{DISCUSSĀO}

O tamanho e a forma da hipófise variam de acordo com a idade, sexo e estado fisiológico do indivíduo (5). A variação de aparência e volume hipofisários também podem ser observados no hipotireoidismo e hipogonadismo primários (6). Portanto, diante de um quadro de massa hipofisária, devemos considerar todas as variantes fisiológicas e patológicas, a fim de evitar uma interpretação diagnóstica equivocada.

A forma mais comum de tumor pituitário é o prolactinoma, o qual apresenta-se com hiperprolactinemia, galactorréia e aumento pituitário (7). O aumento pituitário no contexto do hipotireoidismo primário tem sido descrito há mais de um século, apresentando aumento da sela túrcica à radiografia em mais de $80 \%$ dos pacientes $(3,8)$. Volumoso pseudotumor pituitário causado pelo hipotireoidismo não tratado tem sido descrito como uma condição rara, ocorrendo principalmente em adultos $(1,2,4,6,9,10-14)$. Há apenas alguns relatos desta condição em crianças (15-19). Shimono e cols. (20) descreveram um caso de aumento hipofisário após a suspensão de hormônio tiroideano por um período de apenas 3 semanas.

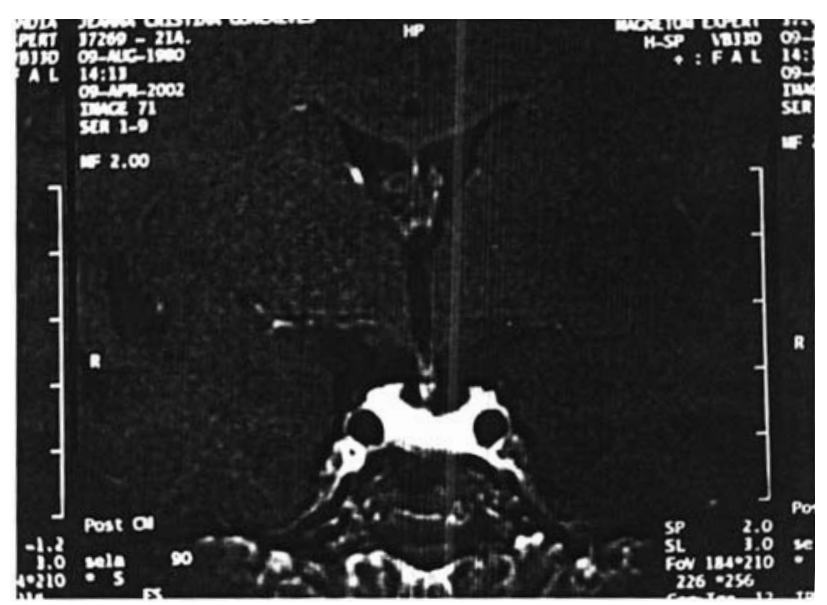

Figura 2. Normalização do volume hipofisário após 2 meses de tratamento do hipotireoidismo com levotiroxina.

Arq Bras Endocrinol Metab vol $48 n^{\circ} 3$ Junho 2004 
Este aumento é geralmente assintomático, entretanto sintomas de massa pituitária como compressão do quiasma óptico, cefaléia, amenorréia e galactorréia, decorrentes de hiperprolactinemia podem estar presentes (11,21). Há inclusive relato de hipertensão intracraniana (17). Sendo, portanto, sempre necessário o diagnóstico diferencial com prolactinoma. No hipotireoidismo primário, a diminuição dos níveis de hormônios tireoideanos circulantes estimula a produção do TRH e TSH, consequentemente levando a uma hiperplasia dos tireotrofos. A causa do aumento dos níveis de prolactina ainda é controversa, tendo o TRH um papel importante estimulando a secreção de prolactina e conseqüentemente hiperplasia dos lactotrofos. Acredita-se que o aumento do tamanho da glândula hipofisária seja decorrente da hiperplasia dos tireotrofos e menos importantemente dos lactotrofos (12).

No caso de nossa paciente, a regressão do volume hipofisário após 2 meses de terapia com levotiroxina confirmou a hipótese diagnóstica de hiperplasia pituitária decorrente do hipotireoidismo primário. O tempo de resposta do tratamento com hormônio tireoideano necessário para regressão da hiperplasia ainda não está bem definido $(13,18,19)$. Há relatos de regressão da massa em 40 dias (19), e ainda há um relato de dramática regressão pituitária com apenas 6 dias de tratamento clínico (13).

Por outro lado, apesar de raro, hipotireoidismo primário e um adenoma hipofisário produtor de prolactina podem ocorrer simultaneamente. Se após a terapia de reposição do hormônio tireoideano, com o paciente eutireoideano, persistirem os sinais clínicos de hiperprolactinemia, podemos ter duas doenças distintas associadas (9).

Relatos de cirurgia transfenoidal para tratamento de massa pituitária confirmado posteriormente como decorrência de hipotireoidismo primário (14) reforçam a importância da avaliação dos hormônios tireoideanos e TSH na investigação de aumento de volume hipofisário prevenindo um tratamento clinico ou cirúrgico inadvertido.

\section{REFERÊNCIAS}

1. Ozbey N, Sariyilidiz E, Yilmaz L, Orhan Y, Sencer E, Molvalilar S. Primary hypothyroidism with hyperprolactinaemia and pituitary enlargement mimicking a pituitary macroadenoma. Int J Clin Pract 1997;51:409-11.

2. Grubb MR, Chakeres D, Malarkey WB. Patients with primary hypothyroidism presenting as prolactinomas. Am J Med 1987;83:765-9.
3. Yamada T, Tsukui T, Ikejiri K, Yukimura Y, Kutani M. Volume of sella turcica in normal subjects and in patients with primary hypothyroidism and hyperthyroidism. J Clin Endocrinol Metab 1976;42:817-22.

4. Young M, Kattner K, Gupta K. Pituitary hyperplasia resulting from primary hypothyroidism mimicking macroadenomas. J Neurosurg 1999;13:138-42.

5. Coronho V, Petroianu A, Santana EM, Pimenta LG. Tratado de endocrinologia e cirurgia endócrina. $1 a$. edição. Guanabara-Koogan:Rio de Janeiro, 2001.

6. Bilaniuk LT, Moshang T, Weingarten MZ, Sutton LN, Samuel LR, Zimmerman RA. Pituitary enlargement mimicking pituitary tumor. J Neurosurg 1985;63:39-42.

7. Von Werder, Klaus. Pituitary investigation. Clin Endocrinol 1996;44:299-303.

8. Niepce B. Enlarged sella due to cretinism (hypothyroidism). In: Ballieri JB, ed. Traitè du goitre et du cretinisme. Paris: 1851.

9. Stokic E, Ivkovic-Lazar T, Lepsanovic L. Hypophyseal pseudomacroadenoma in primary hypothyroidism - 2 case reports. Med Pregl 1992;45:417-9.

10. Mahjoub S, Bouguerra R, Ben Dhia N, Ladeb F, Achour $A$, Elmay $M$, et al. Primary hypothyroidism with hyperprolactinemia and pseudoprolactinoma in 2 sisters. Ann Méd Interne (Paris) 1991;142:311-3.

11. Miranda-Ruiz R, Chavez M, Ruiz-Velasco G, Cstanon J, Zarate A. Primary hypothyroidism associated with a chiasmatic syndrome simulating a prolactinoma. Gac Méd Mex 1990;126:51-4.

12. Khalil A, Kovacs K, Sima AA, Burrow GN, Horvath E. Pituitary thyrotroph hyperplasia mimicking prolactin-secreting adenoma. J Endocrinol Invest 1984;794:399-404.

13. Sarlis NJ, Brucker-Davis F, Doppman JL, Skarulis MC. MRIdemonstrable regression of a pituitary mass in a case of primary hypothyroidism after a week of acute thyroid hormone therapy. J Clin Endocrinol Metab 1997;82:80811.

14. Chan AW, MacFarlane IA, Foy PM, Miles JB. Pituitary enlargement and hyperprolactinaemia due to primary hypothyroidism: errors and delays in diagnoses. J Neurosurg 1990;4:107-12.

15. Ehrim PU, Kerr DS, Cohen AR. Primary hypothyroidism mimicking a pituitary macroadenoma. Pediatr Neurosurg 1998;28:195-7.

16. Papakonstatinou $O$, Bitsori M, Mamoulakis D, Bakantaki A, Papadaki E, Gourtsoyiannis N. MR imaging of pituitary hyperplasia in a child with growth arrest and primary hypothyroidism. Eur Radiol 2000;10:516-8.

17. Adams C, Dean HJ, Israels SJ, Patton A, Fewer DH. Primary hypothyroidism with intracranial hypertension and pituitary hyperplasia. Pediatr Neurol 1994;10:1668.

18. Atchison JA, Lee PA, Albright AL. Reversible suprasellar pituitary mass secondary to hypothyroidism. JAMA 1989;262:3175-7.

19. Kocova M, Netkov S, Sukarova-Angelovska E. Pituitary pseudotumor with unusual presentation reversed short- 
Iy after the introduction of thyroxine replacement therapy. Pediatr Endocrinol Metab 2001;14:1665-9.

20. Shimono T, Hatabu H, Kasagi K, Miki Y, Nishizawa S, Misaki T, et al. Rapid progression of pituitary hyperplasia in humans with primary hypothyroidism: demonstration with MR imaging. Radiology 1999;213:383-8.
21. Yamamoto K, Saito K, Takai T, Naito M, Yoshida S. Visual field defects and pituitary enlargement in primary hypothyroidism. J Clin Endocrinol Metab 1983;57:2837.

Endereço para correspondência:

Carolina de Castro Rocha Betônico

Rua Coriolano Gomes Palmeira 160 apto 92

19013-790 Presidente Prudente, SP 